\title{
Enforcing and Defying Associativity, Commutativity, Totality, and Strong Noninvertibility for One-Way Functions in Complexity Theory
}

\author{
Lane A. Hemaspaandra ${ }^{1}$ \\ Department of Computer Science \\ University of Rochester \\ Rochester, NY 14627, USA \\ Jörg Rothe ${ }^{2}$ \\ Institut für Informatik \\ Heinrich-Heine-Universität Düsseldorf \\ 40225 Düsseldorf, Germany \\ Amitabh Saxena ${ }^{3}$ \\ Department of Computer Science and Computer Engineering \\ La Trobe University \\ Bundoora, VIC 3086, Australia
}

April 21, 2005

\footnotetext{
${ }^{1}$ Supported in part grant NSF-CCF-0426761. Work done in part while visiting JuliusMaximilians-Universität.

${ }^{2}$ Email: rothe@cs.uni-duesseldorf.de. Supported in part by the German Science Foundation (DFG) under grant RO 1202/9-1. Work done in part while visiting the University of Rochester and Julius-Maximilians-Universität.

${ }^{3}$ Email: asaxena@cs.latrobe.edu.au.
} 


\begin{abstract}
Rabi and Sherman [RS97,RS93] proved that the hardness of factoring is a sufficient condition for there to exist one-way functions (i.e., p-time computable, honest, p-time noninvertible functions) that are total, commutative, and associative but not strongly noninvertible. In this paper we improve the sufficient condition to $\mathrm{P} \neq \mathrm{NP}$.

More generally, in this paper we completely characterize which types of one-way functions stand or fall together with (plain) one-way functions - equivalently, stand or fall together with $\mathrm{P} \neq \mathrm{NP}$. We look at the four attributes used in Rabi and Sherman's seminal work on algebraic properties of one-way functions (see [RS97,RS93]) and subsequent papers - strongness (of noninvertibility), totality, commutativity, and associativity - and for each attribute, we allow it to be required to hold, required to fail, or "don't care." In this categorization there are $3^{4}=81$ potential types of one-way functions. We prove that each of these 81 feature-laden types stand or fall together with the existence of (plain) one-way functions.
\end{abstract}

Key words: computational complexity, complexity-theoretic one-way functions, associativity, commutativity, strong noninvertibility. 


\section{Introduction}

\subsection{Motivation}

In this paper, we study properties of one-way functions, i.e., properties of functions that are easy to compute, but hard to invert. One-way functions are important cryptographic primitives and are the key building blocks in many cryptographic protocols. Various models to capture "noninvertibility" and, depending on the model used, various candidates for oneway functions have been proposed. The notion of noninvertibility is usually based on the average-case complexity model in cryptographic applications, whereas noninvertibility for complexity-theoretic one-way functions is usually defined in the worst-case model. Though the average-case model is very important, we note that even the challenge of showing that any type of one-way function exists in the "less challenging" worst-case model remains an open issue after many years of research. It is thus natural to wonder, as a first step, what assumptions are needed to create various types of complexity-theoretic one-way functions. In this paper, we seek to characterize in terms of class separations this existence issue.

Complexity-theoretic one-way functions of various sorts, and related notions, were studied early on by, for example, Berman [Ber77], Brassard, Fortune, and Hopcroft [BFH78, Bra79], Ko [Ko85], and especially Grollmann and Selman [GS88], and have been much investigated ever since; see, e.g., [AR88,Wat88,HH91,Sel92,RS93,HRW97,RS97,HR99,BHHR99, HR00,HPR01,RH02,FFNR03,HT03,Hom04]. The four properties of one-way functions to be investigated in this paper are strongness, totality, commutativity, and associativity. Intuitively, strong noninvertibility - a notion proposed by Rabi and Sherman [RS97,RS93] and later studied in other papers as well [HR99,HPR01,Hom04]-means that for a two-ary function, given some function value and one of the corresponding arguments, it is hard to determine the other argument. It has been known for decades that one-way functions exist if and only if $\mathrm{P} \neq \mathrm{NP}$. But the Rabi-Sherman paper brought out the natural issue of trying to understand what complexity-theoretic assumptions characterized the existence of one-way functions with certain algebraic properties. Eventually, Hemaspaandra and Rothe [HR99] proved that strong, total, commutative, associative one-way functions exist if and only if $\mathrm{P} \neq \mathrm{NP}$. (As mentioned earlier, one-way functions with these properties are the key building blocks in Rabi, Rivest, and Sherman's cryptographic protocols for secret-key agreement and for digital signatures (see [RS97,RS93]).)

This paper provides a detailed study of the four properties of one-way functions mentioned above. For each possible combination of possessing, not possessing, and being oblivious to possession of the property, we study the question of whether such one-way functions can exist. Why should one be interested in knowing if a one-way function possesses "negative" properties, such as noncommutativity? On one hand, negative properties can also have useful applications. For example, Saxena and Soh [SS03,SS04] propose group cryptosystems and authentication protocols for mobile agents with signature chaining that use as their key building blocks strong, associative one-way functions for which commutativity in fact is a disadvantage. More generally, it seems natural to try to catalog which types of one-way functions are created by, for example, simply assuming $\mathrm{P} \neq \mathrm{NP}$. 


\begin{tabular}{|c||c|l|}
\hline $\begin{array}{c}\text { Properties } \\
(s, t, c, a)\end{array}$ & Characterization & \multicolumn{1}{|c|}{ References/Comments } \\
\hline \hline$(\mathrm{N}, \mathrm{N}, \mathrm{N}, \mathrm{N})$ & $\mathrm{P} \neq \mathrm{NP}$ & Lemma $5.2+$ Lemma 3.4 \\
$(\mathrm{~N}, \mathrm{~N}, \mathrm{~N}, \mathrm{Y})$ & $\mathrm{P} \neq \mathrm{NP}$ & Lemma $5.5+$ Lemma 3.4 \\
$(\mathrm{~N}, \mathrm{~N}, \mathrm{Y}, \mathrm{N})$ & $\mathrm{P} \neq \mathrm{NP}$ & Lemma $5.1+$ Lemma 3.4 \\
$(\mathrm{~N}, \mathrm{~N}, \mathrm{Y}, \mathrm{Y})$ & $\mathrm{P} \neq \mathrm{NP}$ & Lemma $5.5+$ Lemma 3.4 \\
\hline$(\mathrm{N}, \mathrm{Y}, \mathrm{N}, \mathrm{N})$ & $\mathrm{P} \neq \mathrm{NP}$ & [HPR01]; see also Lemma 5.2 \\
$(\mathrm{~N}, \mathrm{Y}, \mathrm{N}, \mathrm{Y})$ & $\mathrm{P} \neq \mathrm{NP}$ & Lemma 5.5 \\
$(\mathrm{~N}, \mathrm{Y}, \mathrm{Y}, \mathrm{N})$ & $\mathrm{P} \neq \mathrm{NP}$ & Lemma 5.1 \\
$(\mathrm{~N}, \mathrm{Y}, \mathrm{Y}, \mathrm{Y})$ & $\mathrm{P} \neq \mathrm{NP}$ & Lemma 5.5 \\
\hline$(\mathrm{Y}, \mathrm{N}, \mathrm{N}, \mathrm{N})$ & $\mathrm{P} \neq \mathrm{NP}$ & Lemma $4.5+$ Lemma 3.4 \\
$(\mathrm{Y}, \mathrm{N}, \mathrm{N}, \mathrm{Y})$ & $\mathrm{P} \neq \mathrm{NP}$ & Lemma $4.4+$ Lemma 3.4 \\
$(\mathrm{Y}, \mathrm{N}, \mathrm{Y}, \mathrm{N})$ & $\mathrm{P} \neq \mathrm{NP}$ & Lemma $4.3+$ Lemma 3.4 \\
$(\mathrm{Y}, \mathrm{N}, \mathrm{Y}, \mathrm{Y})$ & $\mathrm{P} \neq \mathrm{NP}$ & Lemma $4.2+$ Lemma 3.4 \\
\hline$(\mathrm{Y}, \mathrm{Y}, \mathrm{N}, \mathrm{N})$ & $\mathrm{P} \neq \mathrm{NP}$ & Lemma 4.5 \\
$(\mathrm{Y}, \mathrm{Y}, \mathrm{N}, \mathrm{Y})$ & $\mathrm{P} \neq \mathrm{NP}$ & Lemma 4.4 \\
$(\mathrm{Y}, \mathrm{Y}, \mathrm{Y}, \mathrm{N})$ & $\mathrm{P} \neq \mathrm{NP}$ & Lemma 4.3 \\
$(\mathrm{Y}, \mathrm{Y}, \mathrm{Y}, \mathrm{Y})$ & $\mathrm{P} \neq \mathrm{NP}$ & [HR99]; see also Lemma 4.2 \\
\hline
\end{tabular}

Table 1: Summary of results.

\subsection{Summary of Our Results}

This paper is organized as follows. In Sections 2 and 3, we formally define the notions and notation used, and we provide some basic lemmas that allow us to drastically reduce the number of cases we have to consider. We will state the full definitions later, but stated merely intuitively, a function is said to be strongly noninvertible if given the output and one argument one cannot efficiently find a corresponding other argument; and a function is said to be strong if it is polynomial-time computable, strongly noninvertible, and satisfies the natural honesty condition related to strong noninvertibility (so-called s-honesty). In Section 4 , we prove that the condition $\mathrm{P} \neq \mathrm{NP}$ characterizes all 27 cases induced by oneway functions that are strong. As a corollary, we also obtain a $\mathrm{P} \neq \mathrm{NP}$ characterization of all 27 cases where one requires one-way-ness but is oblivious to whether or not the functions are strong. In Section 5, we consider functions that are required to be one-way but to not be strong. We show that $\mathrm{P} \neq \mathrm{NP}$ characterizes all of these 27 cases. Thus, $\mathrm{P} \neq \mathrm{NP}$ characterizes all 81 cases overall.

Table 1 summarizes our results for the 16 key cases in which each of the four properties considered is either enforced or defied. ${ }^{1}$ Definition 2.4 provides the classification scheme used in this table. The left column of Table 1 has 16 quadruples of the form $(s, t, c, a)$, where $s$ regards "strong,", $t$ means "total," $c$ means "commutative," and a means "associative."

\footnotetext{
${ }^{1}$ In light of the forthcoming Lemma 3.2 , those cases in which one is oblivious to whether some property holds follow immediately from the cases stated in Table 1.
} 
The variables $s, t, c$, and $a$ take on a value from $\{\mathrm{Y}, \mathrm{N}\}$, where $\mathrm{Y}$ means presence (i.e., "yes"), and $\mathrm{N}$ means absence (i.e., "no") of the given property. The center column of Table 1 states the conditions characterizing the existence of $(s, t, c, a)$-OWFs, and the right column of Table 1 gives the references to the proofs of the results stated.

\subsection{General Proof Strategy}

We do not attempt to brute-force all 81 cases. Rather, we seek to turn the cases' structure and connectedness against themselves. So, in Section 3 we will reduce the 81 cases to their 16 key cases that do not contain "don't care" conditions. Then, also in Section 3, we will show how to derive the nontotal cases from the total cases, thus further reducing our problem to 8 key cases.

As Corollary 4.6 and, especially, much of Section 5 will show, even among the 8 key cases we share attacks, and find and exploit implications.

Thus, the proof in general consists both of specific constructions-concrete (and in some cases rather difficult to discover) realizations forcing for the first time given patterns of properties - and the framework that minimizes the number of such constructions needed.

\section{Preliminaries and Notations}

Fix the alphabet $\Sigma=\{0,1\}$. The set of strings over $\Sigma$ is denoted by $\Sigma^{*}$. Let $\varepsilon$ denote the empty string. Let $\Sigma^{+}=\Sigma^{*}-\{\varepsilon\}$. For any string $x \in \Sigma^{*}$, let $|x|$ denote the length of $x$. Let $\langle\cdot, \cdot\rangle: \Sigma^{*} \times \Sigma^{*} \rightarrow \Sigma^{*}$ be some standard pairing function, that is, some total, polynomial-time computable bijection that has polynomial-time computable inverses and is nondecreasing in each argument when the other argument is fixed. Let nat : $\Sigma^{*} \rightarrow \mathbb{N}$ be the standard bijection between $\Sigma^{*}$ and $\mathbb{N}$, the set of nonnegative integers, i.e., nat maps the lexicographically $i$ th string in $\Sigma^{*}$ to the integer $i-1$. Let FP denote the class of polynomialtime computable functions (this includes both total and nontotal functions). This paper focuses completely on mappings from $\Sigma^{*} \times \Sigma^{*}$ to $\Sigma^{*}$ (they are allowed to be many-to-one and they are allowed to be nontotal, i.e., they may map many distinct pairs of strings from $\Sigma^{*} \times \Sigma^{*}$ to one and the same string in $\Sigma^{*}$, and they need not be defined for all pairs in $\Sigma^{*} \times \Sigma^{*}$ ). (The study of 2-argument one-way functions of course is needed if associativity and commutativity are to be studied.) For each function $f$, let domain $(f)$ denote the set of input pairs on which $f$ is defined, and denote the image of $f$ by image $(f)$.

Definition 2.1 presents the standard notion of a (complexity-theoretic, many-one) oneway function, suitably tailored to the case of two-ary functions in the standard way; see [RS93,RS97,HR99,HPR01,Hom04]. Our one-way functions are based on noninvertibility in the worst-case model, as opposed to noninvertibility in the average-case model that is more appealing for cryptographic applications. The notion of honesty in Definition 2.1 below is needed in order to preclude functions from being noninvertible simply due to the trivial reason that some family of images lacks polynomially short preimages. 
Definition 2.1 (One-Way Function) Let $\sigma$ be a function (it may be either total or nontotal) mapping from $\Sigma^{*} \times \Sigma^{*}$ to $\Sigma^{*}$.

1. We say $\sigma$ is honest if and only if there exists a polynomial $p$ such that for each $z \in$ image $(\sigma)$, there exists a pair $(x, y) \in \operatorname{domain}(\sigma)$ such that $\sigma(x, y)=z$ and $|x|+|y| \leq p(|z|)$.

2. We say $\sigma$ is (polynomial-time) noninvertible if and only if there exists no function $f$ in $\mathrm{FP}$ such that for all $z \in$ image $(\rho)$, we have $\sigma(f(z))=z$.

3. We say $\sigma$ is a one-way function if and only if $\sigma$ is polynomial-time computable, honest, and noninvertible.

The four properties of one-way functions that we will study in this paper are strongness, totality, commutativity, and associativity. A function $\sigma$ mapping from $\Sigma^{*} \times \Sigma^{*}$ to $\Sigma^{*}$ is said to be total if and only if $\sigma$ is defined for each pair in $\Sigma^{*} \times \Sigma^{*}$, and is said to be nontotal if it is not total. We say that a function is partial if it is either total or nontotal; this says nothing, but makes it clear that we are not demanding that the function be total.

We now define the remaining three properties. Rabi, Rivest, and Sherman (see [RS97, RS93]) introduced the notion of strongly noninvertible associative one-way functions (strong AOWFs, for short). Rivest and Sherman (as attributed in [RS97,RS93]) designed cryptographic protocols for two-party secret-key agreement and Rabi and Sherman designed cryptographic protocols for digital signatures, both of which need strong, total AOWFs as their key building blocks. They also sketch protocols for multiparty secret-key agreement that required strong, total, commutative AOWFs. Strong (and sometimes total and commutative) AOWFs have been intensely studied in [HR99,BHHR99,HPR01,Hom04].

Though Rabi and Sherman's [RS97] notion of associativity is meaningful for total functions, it is not meaningful for nontotal two-ary functions, as has been noted and discussed in [HR99]. Thus, we here follow Hemaspaandra and Rothe's [HR99] notion of associativity, which is appropriate for both total and nontotal two-ary functions, and is designed as an analog to Kleene's 1952 [Kle52] notion of complete equality of partial functions.

Definition 2.2 (Associativity and Commutativity) Let $\sigma$ be any partial function mapping from $\Sigma^{*} \times \Sigma^{*}$ to $\Sigma^{*}$. Extend $\Sigma^{*}$ by $\Gamma=\Sigma^{*} \cup\{\perp\}$, where $\perp$ is a special symbol indicating, in the usage " $\sigma(x, y)=\perp$," that $\sigma$ is not defined for the pair $(x, y)$. Define an extension $\widehat{\sigma}$ of $\sigma$, which maps from $\Gamma \times \Gamma$ to $\Gamma$, as follows:

$$
\widehat{\sigma}(x, y)= \begin{cases}\sigma(x, y) & \text { if } x \neq \perp \text { and } y \neq \perp \text { and }(x, y) \in \operatorname{domain}(\sigma) \\ \perp & \text { otherwise. }\end{cases}
$$

1. We say $\sigma$ is associative if and only if for each $x, y, z \in \Sigma^{*}, \widehat{\sigma}(\widehat{\sigma}(x, y), z)=\widehat{\sigma}(x, \widehat{\sigma}(y, z))$.

2. We say $\sigma$ is commutative if and only if for each $x, y \in \Sigma^{*}, \widehat{\sigma}(x, y)=\widehat{\sigma}(y, x)$. 
Informally speaking, strong noninvertibility (see [RS97,RS93]) means that even if a function value and one of the corresponding two arguments are given, it is hard to compute the other argument. It is known that, unless $\mathrm{P}=\mathrm{NP}$, some noninvertible functions are not strongly noninvertible [HPR01]. And, perhaps counterintuitively, it is known that, unless $\mathrm{P}=\mathrm{NP}$, some strongly noninvertible functions are not noninvertible [HPR01]. That is, unless $\mathrm{P}=\mathrm{NP}$, strong noninvertibility does not imply noninvertibility. Strong noninvertibility requires a variation of honesty that is dubbed s-honesty in [HPR01]. The notion defined now, as "strong (function)" in definition 2.3, is in the literature typically called a "strong one-way function." This is quite natural. However, to avoid any possibility of confusion as to when we refer to that and when we refer to the notion of a "one-way function" (see Definition 2.1; as will be mentioned later, neither of these notions necessarily implies the other), we will throughout this paper simply call the notion below "strong" or "a strong function," rather than "strong one-way function."

Definition 2.3 (Strong Function) Let $\sigma$ be any partial function mapping from $\Sigma^{*} \times \Sigma^{*}$ to $\Sigma^{*}$.

1. We say $\sigma$ is s-honest if and only if there exists a polynomial $p$ such that the following two conditions are true:

(a) For each $x, z \in \Sigma^{*}$ with $\sigma(x, y)=z$ for some $y \in \Sigma^{*}$, there exists some string $\hat{y} \in \Sigma^{*}$ such that $\sigma(x, \hat{y})=z$ and $|\hat{y}| \leq p(|x|+|z|)$.

(b) For each $y, z \in \Sigma^{*}$ with $\sigma(x, y)=z$ for some $x \in \Sigma^{*}$, there exists some string $\hat{x} \in \Sigma^{*}$ such that $\sigma(\hat{x}, y)=z$ and $|\hat{x}| \leq p(|y|+|z|)$.

2. We say $\sigma$ is (polynomial-time) invertible with respect to the first argument if and only if there exists an inverter $g_{1} \in \mathrm{FP}$ such that for every string $z \in \operatorname{image}(\sigma)$ and for all $x, y \in \Sigma^{*}$ with $(x, y) \in \operatorname{domain}(\sigma)$ and $\sigma(x, y)=z$,

$$
\sigma\left(x, g_{1}(\langle x, z\rangle)\right)=z .
$$

3. We say $\sigma$ is (polynomial-time) invertible with respect to the second argument if and only if there exists an inverter $g_{2} \in \mathrm{FP}$ such that for every string $z \in \operatorname{image}(\sigma)$ and for all $x, y \in \Sigma^{*}$ with $(x, y) \in \operatorname{domain}(\sigma)$ and $\sigma(x, y)=z$,

$$
\sigma\left(g_{2}(\langle y, z\rangle), y\right)=z \text {. }
$$

4. We say $\sigma$ is strongly noninvertible if and only if $\sigma$ is neither invertible with respect to the first argument nor invertible with respect to the second argument.

5. We say $\sigma$ is strong if and only if $\sigma$ is polynomial-time computable, s-honest, and strongly noninvertible. 
In this paper, we will look at the $3^{4}=81$ categories of one-way functions that one can get by requiring the properties strong/total/commutative/associative to either: hold, fail, or "don't care." For each, we will try to characterize whether such one-way functions exist.

We now define a classification scheme suitable to capture all possible combinations of these four properties of one-way functions.

\section{Definition 2.4 (Classification Scheme for One-Way Functions)}

For each $s, t, c, a \in\{\mathrm{Y}, \mathrm{N}, *\}$, we say that a partial function $\sigma: \Sigma^{*} \times \Sigma^{*} \rightarrow \Sigma^{*}$ is an $(s, t, c, a)$ one-way function (an $(s, t, c, a)$-OWF, for short) if and only if

1. $\sigma$ is a one-way function,

2. if $s=\mathrm{Y}$ then $\sigma$ is strong,

3. if $s=\mathrm{N}$ then $\sigma$ is not strong,

4. if $t=\mathrm{Y}$ then $\sigma$ is a total function,

5. if $t=\mathrm{N}$ then $\sigma$ is a nontotal function,

6. if $c=\mathrm{Y}$ then $\sigma$ is a commutative function,

7. if $c=\mathrm{N}$ then $\sigma$ is a noncommutative function,

8. if $a=\mathrm{Y}$ then $\sigma$ is an associative function, and

9. if $a=\mathrm{N}$ then $\sigma$ is a nonassociative function.

For example, a function is a (Y, Y, Y, Y)-OWF exactly if it is a strong, total, commutative, associative one-way function. And note that, under this definition, whenever a setting is $*$, we don't place any restriction as to whether the corresponding property holds or fails to hold - that is, * is a "don't care" designator. For example, a function is a $(*, \mathrm{Y}, *, *)$-OWF exactly if it is a total one-way function. Of course, all (Y,Y,Y,Y)-OWFs are $(*, \mathrm{Y}, *, *)$ OWFs. That is, our 81 classes do not seek to partition, but rather to allow all possible simultaneous settings and "don't care"s for these four properties. However, the 16 such classes with no stars are certainly pairwise disjoint.

\section{Groundwork: Reducing the Cases}

In this section, we show how to tackle our ultimate goal, stated as Goal 3.1 below, by drastically reducing the number of cases that are relevant among the 81 possible cases.

Goal 3.1 For each $s, t, c, a \in\{\mathrm{Y}, \mathrm{N}, *\}$, characterize the existence of $(s, t, c, a)$-OWF $s$ in terms of some suitable complexity-theoretic condition. 
Since $*$ is a "don't care," for a given $*$ position the characterization that holds with that * is simply the "or" of the characterizations that hold with each of $\mathrm{Y}$ and $\mathrm{N}$ substituted for the $*$. For example, clearly there exist $(\mathrm{Y}, \mathrm{Y}, \mathrm{Y}, *)-\mathrm{OWF}$ if and only if either there exist $(\mathrm{Y}, \mathrm{Y}, \mathrm{Y}, \mathrm{Y})$-OWFs or there exist $(\mathrm{Y}, \mathrm{Y}, \mathrm{Y}, \mathrm{N})$-OWFs. And cases with more than one $*$ can be "unwound" by repeating this. So, to characterize all 81 cases, it suffices to characterize the 16 cases stated in Table 1.

Lemma 3.2 1. For each $t, c, a \in\{\mathrm{Y}, \mathrm{N}, *\}$, there exist $(*, t, c, a)-\mathrm{OWF} s$ if and only if either there exist $(\mathrm{Y}, t, c, a)-\mathrm{OWF} s$ or there exist $(\mathrm{N}, t, c, a)$-OWF $s$.

2. For each $s, c, a \in\{\mathrm{Y}, \mathrm{N}, *\}$, there exist $(s, *, c, a)-\mathrm{OWF} s$ if and only if either there exist $(s, \mathrm{Y}, c, a)-\mathrm{OWF} s$ or there exist $(s, \mathrm{~N}, c, a)-\mathrm{OWF} s$.

3. For each $s, t, a \in\{\mathrm{Y}, \mathrm{N}, *\}$, there exist $(s, t, *, a)-\mathrm{OWF} s$ if and only if either there exist $(s, t, \mathrm{Y}, a)$-OWF $s$ or there exist $(s, t, \mathrm{~N}, a)$-OWF $s$.

4. For each $s, t, c \in\{\mathrm{Y}, \mathrm{N}, *\}$, there exist $(s, t, c, *)-\mathrm{OWF} s$ if and only if either there exist $(s, t, c, \mathrm{Y})$-OWF $s$ or there exist $(s, t, c, \mathrm{~N})$-OWF $s$.

It is well known (see [BDG95] and Proposition 1 of [Sel92]) that $\mathrm{P} \neq \mathrm{NP}$ if and only if $(*, *, *, *)$-OWFs exist, i.e., $\mathrm{P} \neq \mathrm{NP}$ if and only if there exist one-way functions, regardless of whether or not they possess any of the four properties. So, in the upcoming proofs, we will often focus on just showing that $\mathrm{P} \neq \mathrm{NP}$ implies the given type of OWF exists (and we will succeed in 14 of the 16 cases, and 78 of the 81 cases).

Lemma 3.3 For each $s, t, c, a \in\{\mathrm{Y}, \mathrm{N}, *\}$, if there are $(s, t, c, a)$-OWF $s$ then $\mathrm{P} \neq \mathrm{NP}$.

Next, we show that all cases involving nontotal one-way functions can be easily reduced to the corresponding cases involving total one-way functions. Thus, we have eliminated the eight "nontotal" of the remaining 16 cases, provided we can solve the eight "total" cases.

Lemma 3.4 For each $s, c, a \in\{\mathrm{Y}, \mathrm{N}\}$, if there exists an $(s, \mathrm{Y}, c, a)$-OWF, then there exists an $(s, \mathrm{~N}, c, a)-\mathrm{OWF}$.

Proof. Fix any $s, c, a \in\{\mathrm{Y}, \mathrm{N}\}$, and let $\sigma$ be any given $(s, \mathrm{Y}, c, a)$-OWF. For each string $w \in \Sigma^{*}$, let $w^{+}$denote the successor of $w$ in the standard lexicographic ordering of $\Sigma^{*}$, and for each string $w \in \Sigma^{+}$, let $w^{-}$denote the predecessor of $w$ in the standard lexicographic ordering of $\Sigma^{*}$.

Define a function $\rho: \Sigma^{*} \times \Sigma^{*} \rightarrow \Sigma^{*}$ by

$$
\rho(x, y)= \begin{cases}\left(\sigma\left(x^{-}, y^{-}\right)\right)^{+} & \text {if } x \neq \varepsilon \neq y \\ \text { undefined } & \text { otherwise. }\end{cases}
$$

Note that $\rho$ is nontotal, since it is not defined on the pair $(\varepsilon, \varepsilon)$. It is a matter of routine to check that $\rho$ is a one-way function, i.e., polynomial-time computable, honest, and noninvertible. It remains to show that $\rho$ inherits all the other properties from $\sigma$ as well. To this end, we show the following claim. 
Claim 3.5 1. $\sigma$ is commutative if and only if $\rho$ is commutative.

2. $\sigma$ is associative if and only if $\rho$ is associative.

3. $\sigma$ is strong if and only if $\rho$ is strong.

Proof of Claim 3.5 We check these properties separately.

1. Commutativity: Suppose that $\sigma$ is commutative. Given any strings $x, y \in \Sigma^{*}$, if $x=\varepsilon$ or $y=\varepsilon$, then both $\rho(x, y)$ and $\rho(y, x)$ are undefined. If $x \neq \varepsilon \neq y$, then the commutativity of $\sigma$ implies that

$$
\rho(x, y)=\left(\sigma\left(x^{-}, y^{-}\right)\right)^{+}=\left(\sigma\left(y^{-}, x^{-}\right)\right)^{+}=\rho(y, x) .
$$

So $\widehat{\rho}(x, y)=\widehat{\rho}(y, x)$. By Definition 2.2, $\rho$ is commutative.

Conversely, suppose that $\sigma$ is noncommutative. Since $\sigma$ is total, we don't have to worry about holes in the domain of $\sigma$. Let $a$ and $b$ be fixed strings in $\Sigma^{*}$ such that $\sigma(a, b) \neq \sigma(b, a)$. It follows that

$$
\rho\left(a^{+}, b^{+}\right) \neq \rho\left(b^{+}, a^{+}\right) .
$$

Thus, $\rho$ is noncommutative.

2. Associativity: Suppose that $\sigma$ is associative. Let $x, y$, and $z$ be any strings in $\Sigma^{*}$. If $x=\varepsilon$ or $y=\varepsilon$ or $z=\varepsilon$, then both $\rho(x, \rho(y, z))$ and $\rho(\rho(x, y), z)$ are undefined. If none of $x, y$, and $z$ equals the empty string, then the associativity of $\sigma$ implies

$$
\begin{aligned}
\rho(x, \rho(y, z)) & =\left(\sigma\left(x^{-}, \sigma\left(y^{-}, z^{-}\right)\right)\right)^{+} \\
& =\left(\sigma\left(\sigma\left(x^{-}, y^{-}\right), z^{-}\right)\right)^{+} \\
& =\rho(\rho(x, y), z) .
\end{aligned}
$$

So $\widehat{\rho}(x, \widehat{\rho}(y, z))=\widehat{\rho}(\widehat{\rho}(x, y), z)$. By Definition 2.2, $\rho$ is associative.

Conversely, suppose that $\sigma$ is nonassociative. Let $a, b$, and $c$ be fixed strings in $\Sigma^{*}$ such that $\sigma(a, \sigma(b, c)) \neq \sigma(\sigma(a, b), c)$. Since $\sigma$ is total, each of $\sigma(a, b), \sigma(b, c), \sigma(a, \sigma(b, c))$, and $\sigma(\sigma(a, b), c)$ is defined. So

$$
\begin{aligned}
\left(\rho\left(a^{+}, \rho\left(b^{+}, c^{+}\right)\right)\right)^{-} & =\sigma(a, \sigma(b, c)) \\
& \neq \sigma(\sigma(a, b), c) \\
& =\left(\rho\left(\rho\left(a^{+}, b^{+}\right), c^{+}\right)\right)^{-},
\end{aligned}
$$

which implies $\rho\left(a^{+}, \rho\left(b^{+}, c^{+}\right)\right) \neq \rho\left(\rho\left(a^{+}, b^{+}\right), c^{+}\right)$. Thus,

$$
\widehat{\rho}\left(a^{+}, \widehat{\rho}\left(b^{+}, c^{+}\right)\right) \neq \widehat{\rho}\left(\widehat{\rho}\left(a^{+}, b^{+}\right), c^{+}\right) .
$$

By Definition 2.2, $\rho$ is nonassociative. 
3. Strongness: First, we note that $\sigma$ is s-honest if and only if $\rho$ is s-honest. Let $p$ be some polynomial witnessing the s-honesty of $\sigma$ as per Definition 2.3:

(a) For each $x, z \in \Sigma^{*}$ with $\sigma(x, y)=z$ for some $y \in \Sigma^{*}$, there exists some string $\hat{y} \in \Sigma^{*}$ such that $\sigma(x, \hat{y})=z$ and $|\hat{y}| \leq p(|x|+|z|)$.

(b) For each $y, z \in \Sigma^{*}$ with $\sigma(x, y)=z$ for some $x \in \Sigma^{*}$, there exists some string $\hat{x} \in \Sigma^{*}$ such that $\sigma(\hat{x}, y)=z$ and $|\hat{x}| \leq p(|y|+|z|)$.

Since $\rho$ shifts the arguments and the function value of $\sigma$ just by one position in the lexicographic ordering on $\Sigma^{*}$, the polynomial $q(n)=p(n)+1$ witnesses the s-honesty of $\rho$. The converse is proven analogously.

Now, we show that $\sigma$ is strongly noninvertible if and only if $\rho$ is strongly noninvertible. Suppose that $\sigma$ is invertible with respect to the first argument via some inverter $g_{1}$ in FP. That is, for each string $z \in \operatorname{image}(\sigma)$ and for all $x, y \in \Sigma^{*}$ with $(x, y) \in$ domain $(\sigma)$ and $\sigma(x, y)=z$, we have

$$
\sigma\left(x, g_{1}(\langle x, z\rangle)\right)=z
$$

From $g_{1}$ we construct an inverter $f_{1} \in \mathrm{FP}$ that inverts $\rho$ with respect to the first argument as follows. Let $z$ be any string in image $(\rho)$, and let $x, y \in \Sigma^{*}$ be any strings such that $(x, y) \in \operatorname{domain}(\rho)$ and $\rho(x, y)=z$. Given $\langle x, z\rangle, f_{1}$ computes $\left(g_{1}\left(\left\langle x^{-}, z^{-}\right\rangle\right)\right)^{+}$. Note that $\rho$ never maps to the empty string, so $z \neq \varepsilon$ and $z^{-}$is well-defined. Similarly, $x \neq \varepsilon$ because $(x, y) \in \operatorname{domain}(\rho)$, so $x^{-}$is well-defined. Thus,

$$
\rho\left(x, f_{1}(\langle x, z\rangle)\right)=\rho\left(x,\left(g_{1}\left(\left\langle x^{-}, z^{-}\right\rangle\right)\right)^{+}\right)=z .
$$

Similarly, an inverter with respect to the second argument can be built for $\rho$ given one for $\sigma$.

Conversely, given an inverter for $\rho$ with respect to the first (respectively, second) argument, an inverter for $\sigma$ with respect to the first (respectively, second) argument can be constructed by reverting the shifting above. Thus, if $\rho$ is not strongly noninvertible, neither is $\sigma$.

This completes the proof of Lemma 3.4.

Lemmas 3.2, 3.3, and 3.4 imply that it suffices to deal with only the "total" cases. That is, to achieve Goal 3.1, it would be enough to show that if $\mathrm{P} \neq \mathrm{NP}$ then each of the following eight types of one-way functions exist: (Y, Y, Y, Y)-OWFs, (Y, Y, Y, N)OWFs, (Y, Y, N, Y)-OWFs, (Y, Y, N, N)-OWFs, (N, Y, Y, Y)-OWFs, (N, Y, Y, N)-OWFs, $(\mathrm{N}, \mathrm{Y}, \mathrm{N}, \mathrm{Y})-\mathrm{OWF}$, and $(\mathrm{N}, \mathrm{Y}, \mathrm{N}, \mathrm{N})$-OWFs. In the following sections, we will study each of these cases. 


\section{Strongness and Being Oblivious to Strongness: $(\mathrm{Y}, t, c, a)$ - OWFs and $(*, t, c, a)$-OWFs}

In this section, we consider the "strong"-is-required cases and those cases where the property of strongness is a "don't care" issue. We start with the 27 "strong" cases. Theorem 4.1 below characterizes each of these cases by the condition $\mathrm{P} \neq \mathrm{NP}$. The proof of Theorem 4.1 follows from the upcoming Lemmas 4.2 through 4.5, via Lemmas 3.2, 3.3, and 3.4.

Theorem 4.1 For each $t, c, a \in\{\mathrm{Y}, \mathrm{N}, *\}$, there exist $(\mathrm{Y}, t, c, a)-\mathrm{OWF} s$ if and only if $\mathrm{P} \neq$ NP.

Lemma 4.2 is already known from Hemaspaandra and Rothe's work [HR99].

Lemma 4.2 If $\mathrm{P} \neq \mathrm{NP}$ then there exist (Y, Y, Y, Y)-OWFs.

Using Lemmas 3.3 and 4.2, we can exploit the equivalence of $\mathrm{P} \neq \mathrm{NP}$ and the existence of (Y, Y, Y, Y)-OWFs in the upcoming proofs of Lemmas 4.3, 4.4, and 4.5. That is, in these proofs, we start from a strong, total, commutative, associative one-way function.

Lemma 4.3 If $\mathrm{P} \neq \mathrm{NP}$ then there exist (Y, Y, Y, N)-OWF $s$.

Proof. By Lemmas 3.3 and 4.2, the condition $\mathrm{P} \neq \mathrm{NP}$ is equivalent to the existence of some (Y, Y, Y, Y)-OWF, call it $\sigma$. Recall from the proof of Lemma 3.4 that, in the standard lexicographic ordering of $\Sigma^{*}, w^{+}$denotes the successor of $w \in \Sigma^{*}$ and $w^{-}$denotes the predecessor of $w \in \Sigma^{+}$. We use the following shorthand: For $w \in \Sigma^{*}$, let $w^{2+}=\left(w^{+}\right)^{+}$, and for $w \in \Sigma^{*}$ with $w \notin\{\varepsilon, 0\}$, let $w^{2-}=\left(w^{-}\right)^{-}$. Define a function $\rho: \Sigma^{*} \times \Sigma^{*} \rightarrow \Sigma^{*}$ by

$$
\rho(x, y)= \begin{cases}\varepsilon & \text { if } x=y=0 \\ 0 & \text { if } x=y=\varepsilon \\ \varepsilon & \text { if }(x=\varepsilon \wedge y=0) \vee(x=0 \wedge y=\varepsilon) \\ \varepsilon & \text { if }\{x, y\} \cap\{\varepsilon, 0\} \neq \emptyset \wedge\{x, y\} \cap\left(\Sigma^{*}-\{\varepsilon, 0\}\right) \neq \emptyset \\ \left(\sigma\left(x^{2-}, y^{2-}\right)\right)^{2+} & \text { otherwise. }\end{cases}
$$

It is easy to see that $\rho$ is one-way, strong, total, and commutative. This fact can be seen to follow from the construction of $\rho$ and from $\sigma$ having all these properties. However, $\rho$ is not an associative function, since $\rho(\varepsilon, \rho(\varepsilon, 0))=0 \neq \varepsilon=\rho(\rho(\varepsilon, \varepsilon), 0)$.

Thus, $\rho$ is a $(\mathrm{Y}, \mathrm{Y}, \mathrm{Y}, \mathrm{N})-\mathrm{OWF}$.

Lemma 4.4 If $\mathrm{P} \neq \mathrm{NP}$ then there exist (Y, Y, N, Y)-OWF $s$.

Proof. Assuming $\mathrm{P} \neq \mathrm{NP}$. By Lemma 4.2, let $\sigma$ be a (Y,Y,Y,Y)-OWF. Define a function $\rho: \Sigma^{*} \times \Sigma^{*} \rightarrow \Sigma^{*}$ by

$$
\rho(x, y)= \begin{cases}y & \text { if } x, y \in\{0,1\} \\ \left(\sigma\left(x^{3-}, y^{3-}\right)\right)^{3+} & \text { if } x \notin\{\varepsilon, 0,1\} \wedge y \notin\{\varepsilon, 0,1\}) \\ \varepsilon & \text { otherwise }\end{cases}
$$


where we use the following shorthand: Recall from the proof of Lemma 3.4 that, in the standard lexicographic ordering of $\Sigma^{*}, w^{+}$denotes the successor of $w \in \Sigma^{*}$ and $w^{-}$denotes the predecessor of $w \in \Sigma^{+}$. For $w \in \Sigma^{*}$, let $w^{3+}=\left(\left(w^{+}\right)^{+}\right)^{+}$, and for $w \in \Sigma^{*}$ with $w \notin\{\varepsilon, 0,1\}$, let $w^{3-}=\left(\left(w^{-}\right)^{-}\right)^{-}$.

It is easy to see, given the fact that $\sigma$ is a $(\mathrm{Y}, \mathrm{Y}, \mathrm{Y}, \mathrm{Y})-\mathrm{OWF}$, that $\rho$ is a strongly noninvertible, s-honest, total one-way function. However, unlike $\sigma, \rho$ is noncommutative, since

$$
\rho(0,1)=1 \neq 0=\rho(1,0) .
$$

To see that $\rho$, just like $\sigma$, is associative, let three arbitrary strings be given, say $a, b$, and $c$. Distinguish the following cases:

Case 1: Each of $a, b$, and $c$ is a member of $\{0,1\}$. Then, associativity follows from the definition of $\rho$ :

$$
\rho(a, \rho(b, c))=\rho(a, c)=c=\rho(b, c)=\rho(\rho(a, b), c) .
$$

Case 2: None of $a, b$, and $c$ is a member of $\{\varepsilon, 0,1\}$. Then the associativity of $\rho$ follows immediately from the associativity of $\sigma$. That is,

$$
\begin{aligned}
\rho(a, \rho(b, c)) & =\rho\left(a,\left(\sigma\left(b^{3-}, c^{3-}\right)\right)^{3+}\right) \\
& =\left(\sigma\left(a^{3-}, \sigma\left(b^{3-}, c^{3-}\right)\right)\right)^{3+} \\
& =\left(\sigma\left(\sigma\left(a^{3-}, b^{3-}\right), c^{3-}\right)\right)^{3+} \\
& =\rho\left(\left(\sigma\left(a^{3-}, b^{3-}\right)\right)^{3+}, c\right) \\
& =\rho(\rho(a, b), c) .
\end{aligned}
$$

Note here that both $\left(\sigma\left(a^{3-}, b^{3-}\right)\right)^{3+}$ and $\left(\sigma\left(b^{3-}, c^{3-}\right)\right)^{3+}$ are strings that are not members of $\{\varepsilon, 0,1\}$.

Case 3: At least one of $a, b$, and $c$ is not a member of $\{0,1\}$, and at least one of $a, b$, and $c$ is a member of $\{\varepsilon, 0,1\}$. In this case, it follows from the definition of $\rho$ that

$$
\rho(a, \rho(b, c))=\varepsilon=\rho(\rho(a, b), c) .
$$

Thus, $\rho$ is a $(\mathrm{Y}, \mathrm{Y}, \mathrm{N}, \mathrm{Y})-\mathrm{OWF}$.

Lemma 4.5 If $\mathrm{P} \neq \mathrm{NP}$ then there are $(\mathrm{Y}, \mathrm{Y}, \mathrm{N}, \mathrm{N})-\mathrm{OWF} s$.

Proof. Assume P $\neq$ NP. By Lemma 4.2, let $\sigma$ be a (Y, Y, Y, Y)-OWF. Define a function $\rho: \Sigma^{*} \times \Sigma^{*} \rightarrow \Sigma^{*}$ by

$$
\rho(x, y)= \begin{cases}\varepsilon & \text { if } x=y=0 \\ 0 & \text { if } x=y=\varepsilon \\ \varepsilon & \text { if } x=\varepsilon \wedge y=0 \\ 0 & \text { if } x=0 \wedge y=\varepsilon \\ \varepsilon & \text { if }\{x, y\} \cap\{\varepsilon, 0\} \neq \emptyset \wedge\{x, y\} \cap\left(\Sigma^{*}-\{\varepsilon, 0\}\right) \neq \emptyset \\ \left(\sigma\left(x^{2-}, y^{2-}\right)\right)^{2+} & \text { otherwise. }\end{cases}
$$


Again, it follows from the properties of $\sigma$ and the construction of $\rho$ that $\rho$ is one-way, strong, and total. However, $\rho$ is not commutative, since

$$
\rho(\varepsilon, 0)=\varepsilon \neq 0=\rho(0, \varepsilon) .
$$

Furthermore, $\rho$ is not associative, since

$$
\rho(\varepsilon, \rho(\varepsilon, 0))=0 \neq \varepsilon=\rho(\rho(\varepsilon, \varepsilon), 0) .
$$

Thus, $\rho$ is a $(\mathrm{Y}, \mathrm{Y}, \mathrm{N}, \mathrm{N})-\mathrm{OWF}$.

Next, we note Corollary 4.6, which follows immediately from Theorem 4.1 via Lemmas 3.2 and 3.3. That is, in light of Lemmas 3.2 and 3.3, Theorem 4.1 provides also a $\mathrm{P} \neq \mathrm{NP}$ characterization of all 27 cases where one requires one-way-ness but is oblivious to whether or not the functions are guaranteed to be strong.

Corollary 4.6 For each $t, c, a \in\{\mathrm{Y}, \mathrm{N}, *\}$, there are $(*, t, c, a)-\mathrm{OWF} s$ if and only if $\mathrm{P} \neq$ NP.

\section{Nonstrongness: $(\mathrm{N}, t, c, a)-\mathrm{OWFs}$}

It remains to prove the 27 "nonstrong" cases. All 27 have $\mathrm{P} \neq \mathrm{NP}$ as a necessary condition. For each of them, we also completely characterize the existence of such OWFs by $\mathrm{P} \neq \mathrm{NP}$.

First, we consider two "total" and "nonstrong" cases in Lemmas 5.1 and 5.2 below. Note that Hemaspaandra, Pasanen, and Rothe [HPR01] constructed one-way functions that in fact are not strongly noninvertible. Unlike Lemmas 5.1 and 5.2, however, they did not consider associativity and commutativity. Note that, in the proofs of Lemmas 5.1 and 5.2, we achieve "nonstrongness" while ensuring that the functions constructed are shonest. That is, they are not "nonstrong" because they are not s-honest, but rather they are "nonstrong" because they are not strongly noninvertible.

Lemma 5.1 If $\mathrm{P} \neq \mathrm{NP}$ then there exist $(\mathrm{N}, \mathrm{Y}, \mathrm{Y}, \mathrm{N})-\mathrm{OWF} s$.

Proof. Assuming $\mathrm{P} \neq \mathrm{NP}$, we define an (N, Y, Y, N)-OWF that is akin to the one constructed in Theorem 3 of [HPR01]. Define a function $\sigma: \Sigma^{*} \times \Sigma^{*} \rightarrow \Sigma^{*}$ by

$$
\sigma(x, y)= \begin{cases}1 \rho(x) & \text { if } x=y \\ 0 \min (x, y) \max (x, y) & \text { if } x \neq y\end{cases}
$$

where $\min (x, y)$ denotes the lexicographically smaller of $x$ and $y, \max (x, y)$ denotes the lexicographically greater of $x$ and $y$, and $\rho: \Sigma^{*} \rightarrow \Sigma^{*}$ is a total one-ary one-way function, which exists assuming $\mathrm{P} \neq \mathrm{NP}$. Note that $\sigma$ is polynomial-time computable, total, honest, and s-honest. Clearly, if $\sigma$ could be inverted in polynomial time then $\rho$ could be too. Thus, $\sigma$ is a one-way function. However, although $\sigma$ is s-honest, it is not strong. To prove that 
$\sigma$ is not strongly noninvertible, we show that it is invertible with respect to each of its arguments. Define a function $f_{1}: \Sigma^{*} \rightarrow \Sigma^{*}$ by

$$
f_{1}(a)= \begin{cases}y & \text { if }\left(\exists x, y, z \in \Sigma^{*}\right)\left[a=\langle x, 0 z\rangle \wedge z=x y \wedge x<_{\text {lex }} y\right] \\ y & \text { if }\left(\exists x, y, z \in \Sigma^{*}\right)\left[a=\langle x, 0 z\rangle \wedge z=y x \wedge y<_{\text {lex }} x\right] \\ x & \text { if }\left(\exists x, z \in \Sigma^{*}\right)[a=\langle x, 1 z\rangle] \\ \varepsilon & \text { otherwise, }\end{cases}
$$

where $x<_{\text {lex }} y$ indicates that $x$ is strictly smaller than $y$ in the lexicographic ordering of $\Sigma^{*}$. Note that $f_{1}$ is in FP and that $f_{1}$ inverts $\sigma$ with respect to the first argument. Although this is already enough to defy strong noninvertibility of $\sigma$, we note that one can analogously show that $\sigma$ also is invertible with respect to the second argument.

To see that $\sigma$ is commutative, note that if $x=y$ then $\sigma(x, y)=1 \rho(x)=\sigma(y, x)$, and if $x \neq y$ then $\sigma(x, y)=0 \min (x, y) \max (x, y)=\sigma(y, x)$. To see that $\sigma$ is nonassociative, note that $\sigma(\sigma(1,0), 001)=\sigma(001,001)=1 \rho(001) \neq 0100001=\sigma(1,00001)=\sigma(1, \sigma(0,001))$.

Thus, $\sigma$ is an (N, Y, Y, N)-OWF, which completes the proof.

\section{Lemma 5.2 If $\mathrm{P} \neq \mathrm{NP}$ then there exist $(\mathrm{N}, \mathrm{Y}, \mathrm{N}, \mathrm{N})-\mathrm{OWF} s$.}

Proof. Assume that $\mathrm{P} \neq \mathrm{NP}$. So there exists a total one-argument one-way function $\rho: \Sigma^{*} \rightarrow \Sigma^{*}$. In Theorem 3 of [HPR01], a function $\sigma: \Sigma^{*} \times \Sigma^{*} \rightarrow \Sigma^{*}$ is constructed as follows:

$$
\sigma(x, y)= \begin{cases}1 \rho(x) & \text { if } x=y \\ 0 x y & \text { if } x \neq y\end{cases}
$$

It is shown in [HPR01] that $\sigma$ is a total, s-honest one-way function that is not strongly noninvertible.

To see that $\sigma$ is noncommutative, note that

$$
\sigma(0,1)=001 \neq 010=\sigma(1,0)
$$

To see that $\sigma$ is nonassociative, note that

$$
\sigma(\sigma(0,1), 001)=1 \rho(001) \neq 0001001=\sigma(0, \sigma(1,001)) .
$$

Thus, $\sigma$ is an (N, Y, N, N)-OWF, which completes the proof.

Next, we observe that the two remaining "total" and "nonstrong" cases are connected: Lemma 5.3 shows that, given an (N, Y, Y, Y)-OWF, one can construct an (N, Y, N, Y)-OWF. Thus, by Lemma 3.4, characterizing via $\mathrm{P} \neq \mathrm{NP}$ just the case of (N, Y, Y, Y)-OWFs will suffice to solve all the four remaining cases (namely, NYYY, NYNY, NNYY, and NNNY) at once.

Lemma 5.3 If there exist (N, Y, Y, Y)-OWF $s$, then there exist (N, Y, N, Y)-OWFs. 
Proof. The proof uses the construction presented in Lemma 4.4, except that we now start from an (N, Y, Y, Y)-OWF $\sigma$ instead of a (Y, Y, Y, Y)-OWF as in Lemma 4.4. Constructing $\rho$ from $\sigma$ according to the proof of Lemma 4.4 yields a total, noncommutative, associative one-way function that is not strongly noninvertible.

We now turn to completely characterizing the existence of (N, Y, Y, Y)-OWFs. A transformation from the literature that might seem to come close to establishing "if $\mathrm{P} \neq \mathrm{NP}$, then (N, Y, Y, Y)-OWFs exist" has been shown to be flawed unless an unlikely complexity class collapse occurs. ${ }^{2}$ However, the following result of Rabi and Sherman does provide evidence that (N, Y, Y, Y)-OWFs indeed exist.

Theorem 5.4 [RS97,RS93] If factoring is not in polynomial time, then there exist $(\mathrm{N}, \mathrm{Y}, \mathrm{Y}, \mathrm{Y})-\mathrm{OWF} s$.

We now improve that sufficient condition to $\mathrm{P} \neq \mathrm{NP}$.

Lemma 5.5 If $\mathrm{P} \neq \mathrm{NP}$ then there exist $(\mathrm{N}, \mathrm{Y}, \mathrm{Y}, \mathrm{Y})-\mathrm{OWF} s$ and $(\mathrm{N}, \mathrm{Y}, \mathrm{N}, \mathrm{Y})-\mathrm{OWF} s$.

Proof. By Lemma 5.3, it suffices to handle the case of (N, Y, Y, Y)-OWFs. So, assume $\mathrm{P} \neq \mathrm{NP}$. This implies that there exists a total, one-way function $f: \Sigma^{*} \rightarrow \Sigma^{*}$. Define the function $g: \Sigma^{*} \times \Sigma^{*} \rightarrow \Sigma^{*}$ by

$$
g(x, y)= \begin{cases}0 f(a) & \text { if } x=1 a \text { and } y=1 a \\ \varepsilon & \text { otherwise. }\end{cases}
$$

$g$ is clearly a one-way function. $g$ also is clearly total and commutative. $g$ is associative since it is not hard to see that $(\forall a, b, c)[g(a, g(b, c))=g(g(a, b), c)=\varepsilon]$. Though $g$ is easily seen to be s-honest, $g$ fails to be strongly noninvertible, and so is not strong. In particular, given the output and a purported first argument, here is how to find a second argument consistent with the first argument when one exists. If the output is $\varepsilon$ and the purported first argument is $z$, then output $\varepsilon$ as a second argument. If the output is $0 y$ and the purported first argument is $1 x$, then if $f(x)=y$ a good second argument is $1 x$. In every other case, the output and purported first argument cannot have any second argument that is consistent with them, so we safely (though irrelevantly, except for achieving totality of our inverter if one desires that) in this case have our inverter output $\varepsilon$.

\footnotetext{
${ }^{2}$ In more detail: Rabi and Sherman [RS93,RS97], assuming P $\neq$ NP, constructed a nontotal, commutative, associative (in a slightly weaker model of associativity for partial functions that completely coincides with our model when speaking of total functions) one-way function that appears to fail to possess strong noninvertibility. They also proposed a construction that they claim can be used to transform every nontotal AOWF whose domain is in $\mathrm{P}$ to a total AOWF. However, their claim does not provide an (N, Y, Y, Y)-OWF, due to some subtle technical points. First, Rabi and Sherman's construction - even if their claim were validis not applicable to the nonstrong, nontotal, commutative AOWF they construct, since this function seems to not have a domain in P. Second, it it is not at all clear that their above-mentioned "construction to add totality" has the properties they assert for it. In particular, let UP as usual denote Valiant's [Val76] class representing "unambiguous polynomial time." Hemaspaandra and Rothe show in [HR99] that any proof that the Rabi-Sherman claim about their transformation's action is in general valid would immediately prove that $\mathrm{UP}=\mathrm{NP}$, which is considered unlikely.
} 
Theorem 5.6 For each $t, c, a \in\{\mathrm{Y}, \mathrm{N}, *\}$, there exist $(\mathrm{N}, t, c, a)-\mathrm{OWF} s$ if and only if $\mathrm{P} \neq$ NP.

The proof of Theorem 5.6 follows immediately from Lemmas 5.1, 5.2, and 5.5, via Lemmas 3.2, 3.3, and 3.4.

In conclusion, this paper studied the question of whether one-way functions can exist, where one imposes either possession, nonpossession, or being oblivious to possession of the properties of strongness, totality, commutativity, and associativity. We have shown that $\mathrm{P} \neq \mathrm{NP}$ is a necessary and sufficient condition in each of the possible 81 cases.

\section{References}

[AR88] E. Allender and R. Rubinstein. P-printable sets. SIAM Journal on Computing, 17(6):1193-1202, 1988.

[BDG95] J. Balcázar, J. Díaz, and J. Gabarró. Structural Complexity I. EATCS Monographs on Theoretical Computer Science. Springer-Verlag, second edition, 1995.

[Ber77] L. Berman. Polynomial Reducibilities and Complete Sets. PhD thesis, Cornell University, Ithaca, NY, 1977.

[BFH78] G. Brassard, S. Fortune, and J. Hopcroft. A note on cryptography and NP $\cap$ coNP - P. Technical Report TR-338, Department of Computer Science, Cornell University, Ithaca, NY, April 1978.

[BHHR99] A. Beygelzimer, L. Hemaspaandra, C. Homan, and J. Rothe. One-way functions in worst-case cryptography: Algebraic and security properties are on the house. SIGACT News, 30(4):25-40, December 1999.

[Bra79] G. Brassard. A note on the complexity of cryptography. IEEE Transactions on Information Theory, 25(2):232-233, 1979.

[FFNR03] S. Fenner, L. Fortnow, A. Naik, and J. Rogers. Inverting onto functions. Information and Computation, 186(1):90-103, 2003.

[GS88] J. Grollmann and A. Selman. Complexity measures for public-key cryptosystems. SIAM Journal on Computing, 17(2):309-335, 1988.

[HH91] J. Hartmanis and L. Hemachandra. One-way functions and the nonisomorphism of NP-complete sets. Theoretical Computer Science, 81(1):155-163, 1991.

[Hom04] C. Homan. Tight lower bounds on the ambiguity in strong, total, associative, one-way functions. Journal of Computer and System Sciences, 68(3):657-674, 2004 . 
[HPR01] L. Hemaspaandra, K. Pasanen, and J. Rothe. If $\mathrm{P} \neq \mathrm{NP}$ then some strongly noninvertible functions are invertible. In Proceedings of the 13th International Symposium on Fundamentals of Computation Theory, pages 162-171. SpringerVerlag Lecture Notes in Computer Science \#2138, August 2001.

[HR99] L. Hemaspaandra and J. Rothe. Creating strong, total, commutative, associative one-way functions from any one-way function in complexity theory. Journal of Computer and System Sciences, 58(3):648-659, June 1999.

[HR00] L. Hemaspaandra and J. Rothe. Characterizing the existence of one-way permutations. Theoretical Computer Science, 244(1-2):257-261, August 2000.

[HRW97] L. Hemaspaandra, J. Rothe, and G. Wechsung. On sets with easy certificates and the existence of one-way permutations. In Proceedings of the Third Italian Conference on Algorithms and Complexity, pages 264-275. Springer-Verlag Lecture Notes in Computer Science \#1203, March 1997.

[HT03] C. Homan and M. Thakur. One-way permutations and self-witnessing languages. Journal of Computer and System Sciences, 67(3):608-622, 2003.

[Kle52] S. Kleene. Introduction to Metamathematics. D. van Nostrand Company, Inc., New York and Toronto, 1952.

[Ko85] K. Ko. On some natural complete operators. Theoretical Computer Science, 37(1):1-30, 1985.

[RH02] J. Rothe and L. Hemaspaandra. On characterizing the existence of partial oneway permutations. Information Processing Letters, 82(3):165-171, May 2002.

[RS93] M. Rabi and A. Sherman. Associative one-way functions: A new paradigm for secret-key agreement and digital signatures. Technical Report CS-TR3183/UMIACS-TR-93-124, Department of Computer Science, University of Maryland, College Park, Maryland, 1993.

[RS97] M. Rabi and A. Sherman. An observation on associative one-way functions in complexity theory. Information Processing Letters, 64(5):239-244, 1997.

[Sel92] A. Selman. A survey of one-way functions in complexity theory. Mathematical Systems Theory, 25(3):203-221, 1992.

[SS03] A. Saxena and B. Soh. A new paradigm for group cryptosystems using quick keys. In Proceedings of the 11th IEEE International Conference on Networks, pages 385-389. IEEE Computer Society Press, 2003.

[SS04] A. Saxena and B. Soh. An authentication method for mobile agents with signature chaining using non-commutative associative one-way functions. Unpublished manuscript, 2004. 
[Val76] L. Valiant. The relative complexity of checking and evaluating. Information Processing Letters, 5(1):20-23, 1976.

[Wat88] O. Watanabe. On hardness of one-way functions. Information Processing Letters, 27(3):151-157, 1988. 